\title{
An outbreak of foodborne norovirus gastroenteritis linked to a restaurant in Melbourne, Australia, 2014
}

\author{
Shaun P. Coutts, ${ }^{a}$ Kaye Sturge, ${ }^{a}$ Karin Lalor, John A. Marshall, b Leesa D. Bruggink, ${ }^{b}$ Nela Subasinghe ${ }^{c}$ and \\ Marion Easton ${ }^{a}$ \\ Correspondence to Shaun Coutts (email: shaun.coutts@dhhs.vic.gov.au)
}

\begin{abstract}
Introduction: In May 2014 an outbreak of norovirus occurred among patrons of a restaurant in Melbourne, Australia. Investigations were conducted to identify the infectious agent, mode of transmission and source of illness, and to implement controls to prevent further transmission.
\end{abstract}

Methods: A retrospective case-control study was conducted to test the hypothesis that food served at the restaurant between 9 and 15 May 2014 was the vehicle for infection. A structured questionnaire was used to collect demographic, illness and food exposure data from study participants. To ascertain whether any food handlers had experienced gastroenteritis symptoms and were a possible source of infection, investigators contacted and interviewed staff who had worked at the restaurant between 9 and 16 May 2014.

Results: Forty-six cases (including 16 laboratory-confirmed cases of norovirus) and 49 controls were interviewed and enrolled in the study. Results of the analysis revealed a statistically significant association with illness and consumption of grain salad (OR: 21.6, 95\% Cl: 1.8-252.7, $p=0.015$ ) and beetroot dip (OR: 22.4, 95\% Cl: 1.9-267.0, $p=0.014$ ). An interviewed staff member who reported an onset of acute gastrointestinal illness on 12 May 2014 had prepared salads on the day of onset and the previous two days.

Discussion: The outbreak was likely caused by person-to-food-to-person transmission. The outbreak emphasizes the importance of the exclusion of symptomatic food handlers and strict hand hygiene practices in the food service industry to prevent contamination of ready-to-eat foods and the kitchen environment.

$\mathrm{N}$ oroviruses are non-enveloped, single-stranded RNA viruses, recognized as a leading cause of acute gastroenteritis worldwide. ${ }^{1}$ There are currently six recognized norovirus genogroups, three of which (GI, GIl and GIV) cause human illness. ${ }^{2}$ Norovirus is transmitted via the faecal-oral route primarily through close contact with an infected person, contact with contaminated fomites or consumption of contaminated food or water. ${ }^{3}$ The average incubation period is between 24 and 48 hours, with symptoms including acuteonset vomiting, diarrhoea, nausea, myalgia and lowgrade fever. ${ }^{4}$ Infected individuals shed the virus while symptomatic; however, shedding has been documented before the onset of symptoms, after symptoms have resolved and by asymptomatic infected individuals. ${ }^{5-7}$ Contamination of food by both symptomatic and asymptomatic infected food handlers has been well documented. ${ }^{8-14}$
Commencing 13 May 2014, the Victorian Department of Health and Human Services, Communicable Disease Prevention and Control Unit received reports of gastrointestinal illness in patrons following a banquet lunch at a Mediterranean-style restaurant on 11 May 2014. In response, an outbreak investigation was initiated with the local council health department to identify the infectious agent, the mode of transmission, the source of illness and to implement controls to prevent further transmission.

\section{METHODS}

\section{Epidemiological investigation}

A retrospective case-control study was conducted to test the hypothesis that food served at the restaurant was the vehicle for infection. Study participants were recruited from the restaurant's booking list and contacted for 
phone interviews. A structured questionnaire was used to collect demographic, illness and food exposure data from study participants.

A probable case was defined as a person who ate food at the restaurant between 9 and 15 May 2014 and had onset of vomiting and/or diarrhoea or two or more symptoms of fever, nausea, abdominal pain and headache between 24 and 48 hours after consumption. A confirmed case met the probable case definition and also had norovirus detected by polymerase chain reaction (PCR) in a faecal specimen. Controls were patrons identified during the interview process who did not meet the definition of a probable or confirmed case but had eaten at the restaurant between 9 and 15 May 2014 .

To ascertain whether any food handlers had experienced gastroenteritis symptoms and were a possible source of infection, investigators contacted and interviewed staff who worked at the restaurant between 9 and 16 May 2014.

Data analysis was conducted using Stata 13 (StataCorp, College Station, TX). Univariate analysis was used to calculate $p$-values (2-sided Fisher exact), odds ratios (OR) and $95 \%$ confidence intervals $(\mathrm{Cl})$ for food exposure variables. Variables with a $p$-value $<0.05$ in univariate analysis were included in a multivariable logistic regression model. Backward elimination was used to refine the model with the variable with the highest $p$-value $>0.05$ removed at each elimination step. Variables found to be statistically significant on univariate and multivariable analyses were reported.

\section{Environmental and laboratory investigation}

Environmental health officers from the council health department conducted an environmental investigation at the restaurant. Food samples were obtained and tested for Salmonella spp., coagulase-positive staphylococci, Bacillus cereus and Clostridium perfringens at the Microbiological Diagnostic Unit Public Health Laboratory (MDU PHL). Food samples were not tested for norovirus as the molecular detection of norovirus in food remains prohibitively expensive, time consuming and often unsuccessful because of the heterogeneous distribution of low numbers of virus particles in foods. ${ }^{13}$

Stool specimens were obtained from cases where possible and tested for bacterial enteric pathogens at MDU PHL. The specimens were tested for norovirus by reverse transcription polymerase chain reaction (RT-PCR) at the Victorian Infectious Diseases Reference Laboratory and nucleotide sequencing of norovirus RNA was conducted where appropriate, as previously reported. ${ }^{14}$

\section{Ethics and permissions}

Ethics approval was not sought as the investigation was undertaken as part of a public health response to an outbreak.

\section{RESULTS}

\section{Epidemiological investigation}

Forty-six cases (including 16 confirmed cases) and 49 controls were identified and interviewed. The majority of cases dined at the restaurant on 11 May 2014 with most experiencing an onset of symptoms during the afternoon of 12 May 2014 or the morning of 13 May 2014. The last recorded case ate at the restaurant on 15 May 2014 and had an onset of symptoms on 17 May 2014. An incubation period was recorded for 29 cases, and a median of 28 hours (range 7 to 57 hours) was observed. Symptom duration was recorded for 27 cases, and a median duration of two days (range 0.5 to 5 days) was reported. Symptom characteristics of the cases are described in Table 1.

Twelve staff members who worked between 9 and 16 May were interviewed during the investigation. Only one staff member reported experiencing acute gastroenteritis during this period with an onset of illness on 12 May 2014. The staff member had prepared salads on the day of onset and the previous two days. The staff member was unwilling to provide a faecal specimen for laboratory testing.

In univariate analysis, foods significantly associated with illness were the beetroot dip, grain salad (a cold salad containing freekeh wheat, lentils, parsley and nuts), coleslaw, calamari and lamb cutlets (Table 2). In the final multivariable logistic regression model, two foods remained significantly associated with illness: the grain salad (OR: 21.6, 95\% Cl: 1.8-252.7, $p=0.015$ ) and the beetroot dip (OR: 22.4, 95\% Cl: 1.9-267.0, $p=0.014$ ).

A review of food frequency data revealed 45 cases 
Table 1. Symptom characteristics of 46 cases identified among patrons of a restaurant in Melbourne, Australia, 2014

\begin{tabular}{ccc}
\hline Symptom & Number of cases reporting & Percentage of cases reporting \\
\hline Diarrhoea & 45 & $98 \%$ \\
Abdominal pain & 39 & $85 \%$ \\
Nausea & 33 & $72 \%$ \\
Vomiting & 29 & $63 \%$ \\
Headache & 27 & $59 \%$ \\
Fever & 16 & $35 \%$ \\
\hline
\end{tabular}

Table 2. Food items associated with illness by univariate analysis

\begin{tabular}{ccccc}
\hline Food Item & $\begin{array}{c}\text { Cases } \\
(\mathrm{n}=\mathbf{4 6})(\%)\end{array}$ & $\begin{array}{c}\text { Controls } \\
(\mathrm{n}=\mathbf{4 9 )}(\%)\end{array}$ & $\begin{array}{c}\text { Odds ratio } \\
(\mathbf{9 5 \%} \text { confidence interval) }\end{array}$ & $\boldsymbol{p}$-value \\
\hline Beetroot dip & $11(24)$ & $1(2)$ & $15.1(2.0-662.4)$ & 0.001 \\
Grain salad & $45(98)$ & $37(76)$ & $14.6(2.0-637.3)$ & 0.002 \\
Coleslaw & $18(39)$ & $9(18)$ & $2.9(1.0-8.3)$ & 0.025 \\
Calamari & $9(20)$ & $2(4)$ & $5.7(1.1-56.6)$ & 0.018 \\
Lamb cutlets & $9(20)$ & $2(4)$ & $5.7(1.1-56.6)$ & 0.018 \\
\hline
\end{tabular}

(98\%) recalled eating the grain salad, and one case did not consume any other food from the restaurant except the grain salad. By comparison, only 11 of the 46 cases (24\%) reported consumption of the beetroot dip.

\section{Environmental and laboratory investigation}

Sixteen of the 23 stool specimens were positive for Gll norovirus. Additional studies indicated this was the epidemic strain GII.Pe/Gll.4_Sydney_2012. All food and stool samples tested were negative for bacterial pathogens.

A senior staff member at the restaurant reported the grain salad was generally prepared in batches of approximately 12 kilograms to be used over a period of three to four days. It was mixed in large containers by hand; gloves were not always worn during the mixing process. An unusually large 19 kilogram batch of grain salad was made in preparation for the 11 May 2014 lunch. The salad did not undergo any processing steps (such as cooking) that would have inactivated norovirus potentially introduced by an infected food handler during preparation.

The environmental investigation revealed some of the handwashing facilities in food preparation areas were obstructed and not supplied with soap.

\section{DISCUSSION}

This point source outbreak of norovirus among restaurant patrons is likely to have been caused by person-to-foodto-person transmission. The results of the case-control study analysis, the higher frequency of consumption of the grain salad among cases, the likelihood an infectious food handler prepared this menu item and the ready-toeat nature of the product all support the grain salad as the most likely vehicle of infection, though it is possible that the beetroot dip may have also been contaminated. The large batch size of the grain salad and the extended time over which it was served likely contributed to the protracted onsets of illness among patrons.

Although norovirus infection was not confirmed in any food handlers at the restaurant, it is suspected that ready-to-eat food was contaminated by a food handler during the pre-symptomatic or the early-symptomatic stage of illness. The lack of adequate handwashing facilities in food preparation areas further supports this hypothesis, as does anecdotal evidence that salads were 
often mixed with bare hands. National food standards in Australia require food handlers to take all practical measures to prevent unnecessary contact with ready-to-eat food and detail requirements for the availability and use of handwashing facilities by food handlers. ${ }^{15}$

National food standards in Australia also require a food business to exclude employees having a foodborne disease from handling food until a medical practitioner advises the employee no longer has or is carrying the disease. ${ }^{15}$ While this requirement aims to minimize the risk of food contamination by ill food handlers, it may also discourage reporting of gastroenteritis among food handlers, many of whom are employed on a casual basis and receive no entitlements when absent from work for medical reasons.

Several limitations were identified in this study. The retrospective nature of the case-control study makes it impossible to rule out recall bias, which may have been exacerbated by the large number of food choices available on the banquet menu. The lack of a practical method for the molecular detection of norovirus in food samples meant that it was not possible to confirm the presence of norovirus in the implicated foods. Food handlers, including asymptomatic individuals, were not tested for norovirus infection during the investigation, so the presence of an infected food handler at the premises could not be laboratory-confirmed.

This outbreak emphasizes the importance of the exclusion of symptomatic food handlers and strict hand hygiene practices in the food service industry to prevent contamination of ready-to-eat foods and the kitchen environment. It is essential that food regulators continue to promote and enforce these requirements on food business operators to prevent future outbreaks of norovirus caused by infectious food handlers.

\section{Conflicts of Interest}

None.

Funding

None.

\section{Acknowledgements}

We would like to acknowledge the invaluable assistance provided during this investigation by staff in the Department of Health and Human Services Communicable Disease Prevention and Control, Communicable Disease Epidemiology and Surveillance and East Division teams, Boroondara City Council Health Services, the Microbiological Diagnostic Unit Public Health Laboratory and the Victorian Infectious Diseases Reference Laboratory. Special thanks to Joy Gregory and James Fielding for their guidance on the preparation of the manuscript.

\section{References}

1. Ahmed SM, Hall AJ, Robinson AE, Verhoef L, Premkumar P, Parashar UD, et al. Global prevalence of norovirus in cases of gastroenteritis: a systematic review and meta-analysis. Lancet Infect Dis. 2014 Aug;14(8):725-30. pmid:24981041 doi:10.1016/ S1473-3099(14)70767-4

2. Robilotti E, Deresinski S, Pinsky BA. Norovirus. Clin Microbiol Rev. 2015 Jan;28(1):134-64. pmid:25567225 doi:10.1128/ CMR.00075-14

3. Patel MM, Hall AJ, Vinjé J, Parashar UD. Noroviruses: a comprehensive review. J Clin Virol. 2009 Jan;44(1):1-8. pmid:19084472 doi:10.1016/j.jcv.2008.10.009

4. Matthews JE, Dickey BW, Miller RD, Felzer JR, Dawson BP, Lee AS, et al. The epidemiology of published norovirus outbreaks: a review of risk factors associated with attack rate and genogroup. Epidemiol Infect. 2012 Jul;140(07):1161-72. pmid:22444943 doi:10.1017/S0950268812000234

5. Atmar RL, Opekun AR, Gilger MA, Estes MK, Crawford SE, Neill $\mathrm{FH}$, et al. Norwalk virus shedding after experimental human infection. Emerg Infect Dis. 2008 Oct;14(10):1553-7. pmid:18826818 doi:10.3201/eid1410.080117

6. Rockx B, De Wit M, Vennema H, Vinjé J, De Bruin E, Van Duynhoven $\mathrm{Y}$, et al. Natural history of human calicivirus infection: a prospective cohort study. Clin Infect Dis. 2002 Aug 01;35(3):24653. pmid:12115089 doi:10.1086/341408

7. Teunis PFM, Sukhrie FHA, Vennema H, Bogerman J, Beersma MFC, Koopmans MPG. Shedding of norovirus in symptomatic and asymptomatic infections. Epidemiol Infect. 2015 Jun;143(08):1710-7. pmid:25336060 doi:10.1017/S095026881400274X

8. Ozawa K, Oka T, Takeda N, Hansman GS. Norovirus infections in symptomatic and asymptomatic food handlers in Japan. J Clin Microbiol. 2007 Dec;45(12):3996-4005. pmid:17928420 doi:10.1128/JCM.01516-07

9. Maritschnik S, Kanitz EE, Simons E, Höhne M, Neumann H, Allerberger $F$, et al. A Food Handler-Associated, Foodborne Norovirus Gll.4 Sydney 2012-Outbreak Following a Wedding Dinner, Austria, October 2012. Food Environ Virol. 2013 Sep 12;5(220). pmid:24026524

10. Friedman DS, Heisey-Grove D, Argyros F, Berl E, Nsubuga J, Stiles $\mathrm{T}$, et al. An outbreak of norovirus gastroenteritis associated with wedding cakes. Epidemiol Infect. 2005 Dec;133(06):1057-63. pmid:16274502 doi:10.1017/S0950268805004760 
11. Mayet A, Andreo V, Bedubourg G, Victorion S, Plantec J, Soullie $B$, et al. Food-borne outbreak of norovirus infection in a French military parachuting unit, April 2011. Euro Surveill. 2011 July 28;16(30):19930. pmid:21813082

12. Chen M-Y, Chen W-C, Chen P-C, Hsu S-W, Lo Y-C. An outbreak of norovirus gastroenteritis associated with asymptomatic food handlers in Kinmen, Taiwan. BMC Public Health. 2016 May 04;16(1):372. pmid:27143036 doi:10.1186/s12889-016-3046-5

13. Stals A, Baert L, De Keuckelaere A, Van Coillie E, Uyttendaele $M$. Evaluation of a norovirus detection methodology for ready-to-

eat foods. Int J Food Microbiol. 2011 Feb 28;145(2-3):420-5. pmid:21333370 doi:10.1016/j.ijfoodmicro.2011.01.013

14. Bruggink LD, Dunbar NL, Catton MG, Marshall JA. Norovirus genotype diversity associated with gastroenteritis outbreaks in Victoria in 2013. Commun Dis Intell Q Rep. 2015 March 31;39(1):E3441. pmid:26063096

15. Australia New Zealand Food Standards Code. Canberra, A.C.T: Food Standards Australia New Zealand; 2016 (www.foodstandards.gov.au/code, accessed 28 April 2017). 